\title{
Research on HPM Teaching Supported by Hawgent Dynamic Mathematics Software: Take "The Recognition of Circle" as an Example
}

\author{
Linfeng Han ${ }^{*}$, Qian Tao² \\ ${ }^{1}$ Department of Mathematics and Statistics, Guangxi Normal University, Guilin 541006, Guangxi Zhuang Autonomous \\ Region, China \\ ${ }^{2}$ Chongqing Qianjiang Shijia Primary School, Chongqing 409011, China \\ *Corresponding author: Linfeng Han, hlf.math@foxmail.com

\begin{abstract}
History and Pedagogy of Mathematics (HPM) is one of the important research fields in mathematics education, which has received widespread attention from the mathematics education community because of its educational value. Modern mathematics education technology plays an important auxiliary role in mathematics teaching. Hawgent is a dynamic mathematics software that can present abstract mathematical knowledge visually and static mathematical knowledge dynamically. In view of this, this research takes "the recognition of circle" as an example to conduct a research on HPM teaching supported by Hawgent Dynamic Mathematics Software in three aspects: analyze the contents and uncover the history of mathematics, make the products and show the history of mathematics, design the teaching and integrate the history of Mathematics.
\end{abstract}

Keywords: HPM; Education technology; Hawgent; Mathematics teaching

Publication date: August 2021; Online publication: August 30, 2021

\section{Introduction}

HPM (History and Pedagogy of Mathematics) is a field that studies the relationship between the history of mathematics and mathematics education. The value of integrating the history of mathematics into mathematics teaching mainly includes six categories: presenting the harmony of knowledge, displaying the beauty of methods, creating the pleasure of inquiry, showing the charm of culture, providing the help of ability, and achieving the effect of moral education ${ }^{[1]}$. With the development of information technology, computer-assisted teaching has been widely used in mathematics teaching practice. As a dynamic mathematical software, Hawgent is flexible and convenient to operate, and coupled with powerful mathematical functions and rich dynamic resources ${ }^{[2]}$. Therefore, Hawgent can present abstract mathematical knowledge visually and static mathematical knowledge dynamically. HPM Teaching Supported by Hawgent Dynamic Mathematics Software can visualize and dynamically display the content of the history of mathematics in the classroom, making HPM teaching more vivid and efficient. This research takes "The Recognition of Circle" as an example to practice HPM teaching supported by Hawgent, and hopes to provide some reference for teachers' teaching.

\section{Analysis of the contents of "circle"}

"Circle" is the first plane curve figure learnt by students, and it is also the basis of subsequent learningrelated content, and it is important among plane figures. The Pythagorean School of ancient Greece believes 
that the most beautiful of all plane figures is the circle ${ }^{[3]}$. It can be seen that "circle" embodies rich mathematical beauty and history of mathematics.

\subsection{Ancient application of "circle"}

As people deepen their understanding of "circle," the applications of "circle" are becoming more and more abundant. About 18,000 years ago, cavemen drilled circular holes in animal teeth and other objects. In the age of pottery, most of the pottery made by people was round. About 6,000 years ago, Mesopotamians made the world's first wheel - a round wooden disc. About 4,000 years ago, people fixed the round wooden disc under the wooden frame to make something which became the first car ${ }^{[4]}$. "Circle" also has obvious applications in ancient Chinese architecture. For example, the roof of the Temple of Heaven is round.

\subsection{Ancient theory of "circle"}

More than 2,000 years ago, the Chinese philosopher Mozi gave circle a definition in the Mojing, which states that "a circle has the same length as one middle." "[5] Liu Hui from the Wei and Jin Dynasties of China wrote the "Nine Chapters of Arithmetic" in 263 AD in the Fang Tian Zhang in the note of "round field technique," the circle cutting technique is proposed as the basis for calculating the circumference, area and $\pi$ of a circle ${ }^{[6]}$. Zu Chongzhi continued to calculate on the basis of previous calculations and found that the $\boldsymbol{\pi}$ was between 3.1415926 and 3.1415927. This result made him the first person in the world to accurately determine the value of $\pi$ to the 7 th place after the decimal point.

\section{Making the products of "circle"}

According to the content of "circle" mathematics history, Hawgent dynamic mathematics software is used to make related dynamic products.

\subsection{Making a "Definition of Circle" product}

\subsubsection{Step 1: Draw a circle}

Click "Activate the tool for drawing objects," and click the left mouse button to draw two points O and A at a suitable position on the interface. Hold down the "Ctrl" mouse and click on the two points $\mathrm{O}$ and A, and click "Draw|Circle" (Figure 1).

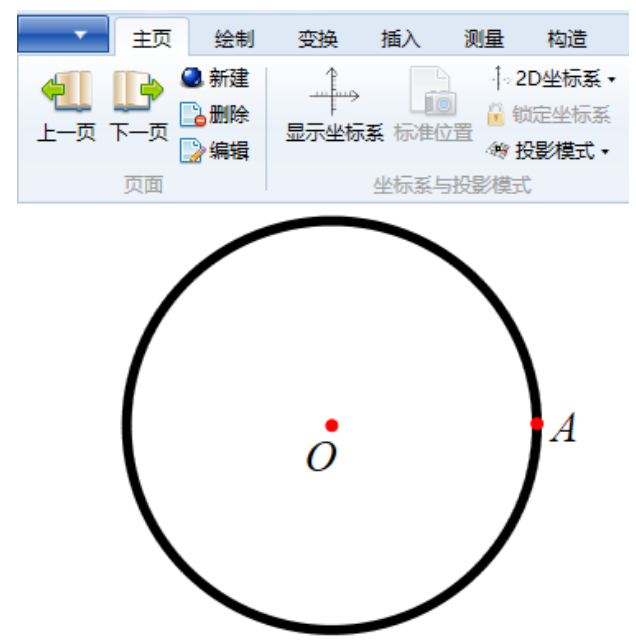

Figure 1. Draw a circle 


\subsubsection{Step 2: Draw point $A$ and rotate one circle}

First, hold down the "Ctrl" mouse and click the object "circle," then click point A, and click "Transform|Rotate." For the pop-up dialog box, enter 360 in the "Rotation Angle" and click "OK." Then, hold down the "Ctrl" mouse and click the object "circle," then click point A, and click "Insert|Animation|Variable." For the pop-up dialog box, enter 360 in the "Maximum" field. Finally, click on point A and then click on "Draw|Track" to hide the object "circle" and click on the "animation" button to present the dynamic process of point A rotating around point $\mathrm{O}$ to form a "circle" (Figure 2).

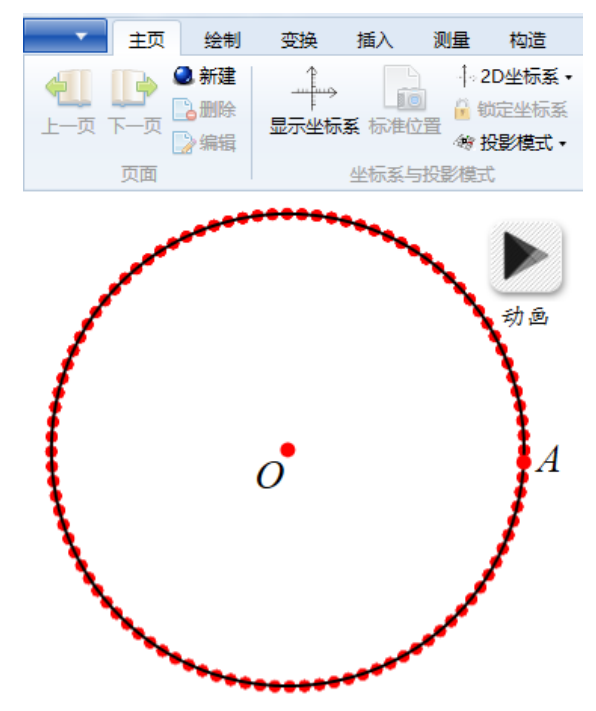

Figure 2. Draw point $A$ and rotate one circle

\subsubsection{Step 3: Draw the radius of the circle}

First, hold down the "Ctrl" mouse and click point O, then point A, and click "Draw|Line Segment." In this way, when point $\mathrm{A}$ is rotating, it will drive the radius OA to rotate, and then add "measurement" to highlight the constant distance from the moving point $A$ to the fixed point $\mathrm{O}$ (Figure 3 ).

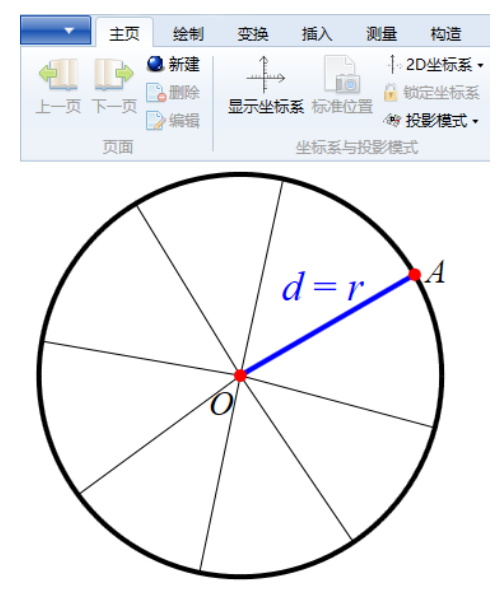

Figure 3. Draw the radius of the circle 


\subsection{Making a "Cut Circle Technique" product}

\subsubsection{Step 1: Draw a circle}

Click "Activate the tool for drawing objects," and click the left mouse button to draw two points O and A at a suitable position on the interface. Hold down the "Ctrl" mouse and click on the two points O and A, and click "Draw|Circle" (Figure 4).

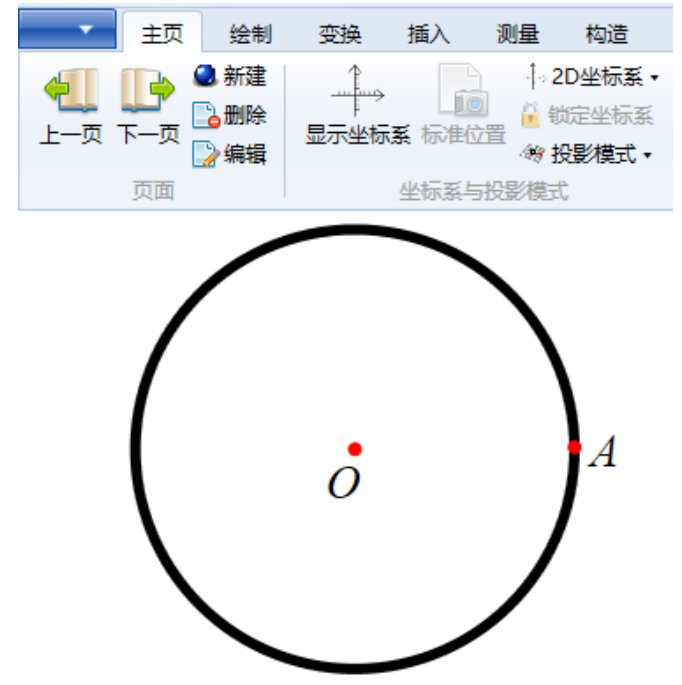

Figure 4. Draw a circle

\subsubsection{Step 2: Draw inscribed regular polygon}

First, click "Insert | Variable Ruler." For the pop-up dialog box, enter a in the "Variable" field, enter 0 in the "Left end," enter 100 in the "Right end," and click "OK." This makes the number of sides of the regular polygon inscribed in the circle range from 0 to 100 . Since the number of edges is required to be a positive integer, click "Measure|Expression" again. For the pop-up dialog box, enter: floor $(a)$ to get the measured variable u000. This makes the number of sides of the regular polygon inscribed in the circle a positive integer in the range of 0 to 100. Finally, hold down the "Ctrl" mouse and click on the circle and point A, click "Draw|Regular Polygon|Inscribed Regular Polygon," for the pop-up dialog box, enter: u000. In this way, the number of sides of the inscribed regular polygon can be changed by dragging the variable ruler. When the number of sides of the inscribed regular polygon increases indefinitely, the shape will be closer to a circle (Figure 5).

\subsection{Make a " $\pi$ " product}

\subsubsection{Step 1: Draw a circle}

Click to display the coordinate system, click "Draw | Coordinate Point." For the pop-up dialog box, enter $a$ in the "x-axis coordinate" and 0.5 in the "y-axis coordinate" to get the point $(a, 0.5)$, and this point is determined as the center coordinate of the circle. Then, click "Insert|Variable Ruler." For the pop-up dialog box, enter $a$ at the minimum value and $\pi$ at the maximum value, and click "OK." Then, hold down the "Ctrl" mouse and click the center coordinates $(a, 0.5)$, click "Draw|Circle." For the dialog box that pop-up, enter 0.5 in the radius. In this way, a circle with center coordinates $(a, 0.5)$ and a radius of 0.5 is drawn, and the value range of $a$ is $[0, \pi]$. Finally, click on the "circle" object, and click "design I line" to turn the circumference of "circle" into dotted line (Figure 6). 


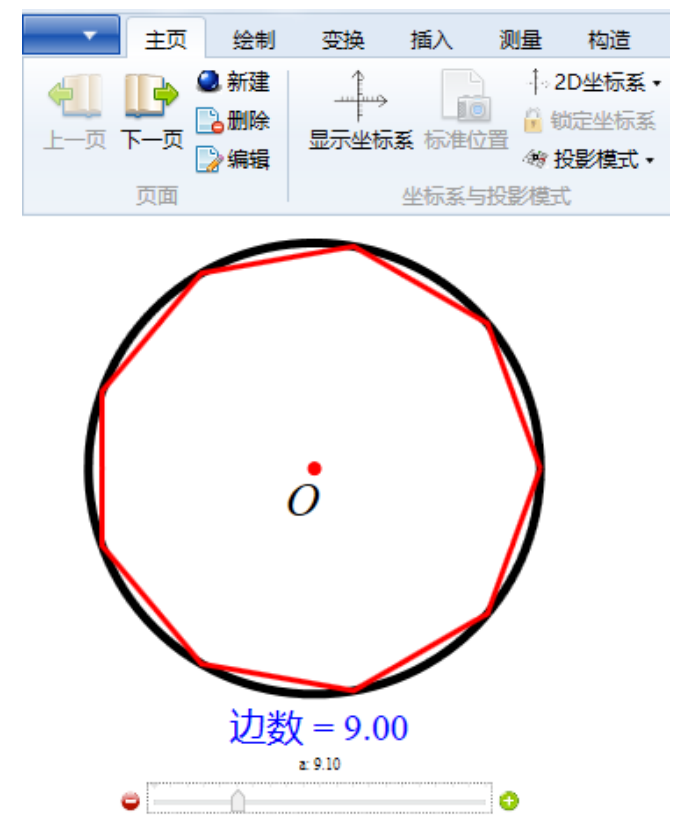

Figure 5. Draw inscribed regular polygon

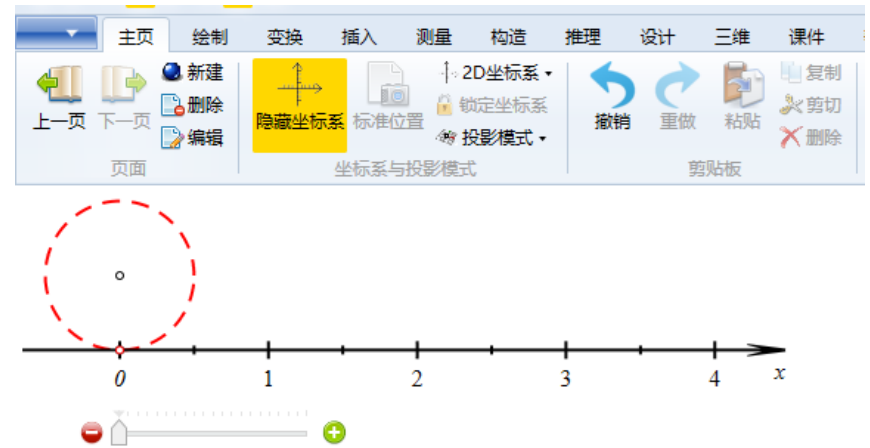

Figure 6. Draw a circle

\subsubsection{Step 2: Draw parametric curve}

First, click "Draw|Coordinate Point." For the pop-up dialog box, enter $a$ in the "x-axis coordinate" and 0 in the "y-axis coordinate" to get the point $(a, 0)$. Click "Draw|Coordinate Point" again. For the pop-up dialog box, enter 0 in the "x-axis coordinate" and 0 in the "y-axis coordinate" to get the point $(0,0)$. Hold down the "Ctrl" mouse and click the point $(0,0)$ first, then click the point $(a, 0)$, and click "Draw|Line Segment." Then, click "Draw $\mid$ Coordinate Point." For the pop-up dialog box, enter " $a+0.5 \cdot \cos (-\pi / 2-2 a)$ " in the "xaxis coordinate," and enter " $0.5+0.5 \cdot \operatorname{Sin}(-\pi / 2-2 a)$ " in the "y-axis coordinate" to get the point $(a+0.5 \cdot \cos (-$ $\pi / 2-2 a), 0.5+0.5 \cdot \sin (-\pi / 2-2 a))$. Finally, click "Draw $\mid$ General Curve $\mid$ Parametric Equation." For the popup dialog box, enter " $a+0.5 \cdot \cos (-\pi / 2+2 \mathrm{t})$ " in the " $x=x(\mathrm{t})$ " field, and in the " $y=y(\mathrm{t})$ " input " $0.5+0.5 \cdot \sin (-$ $\pi / 2+2 t)$ ", input 0 at the "minimum value," and input " $\pi-a$ " at the "maximum value" to obtain the parameter curve. In this way, the "variable ruler" can be dragged to depict the circumference of the circle on the number axis, and the radius of the drawn circle is 0.5 , so the circumference is $\pi$, then the $\pi$ is dynamically depicted on the number axis (Figure 7). 


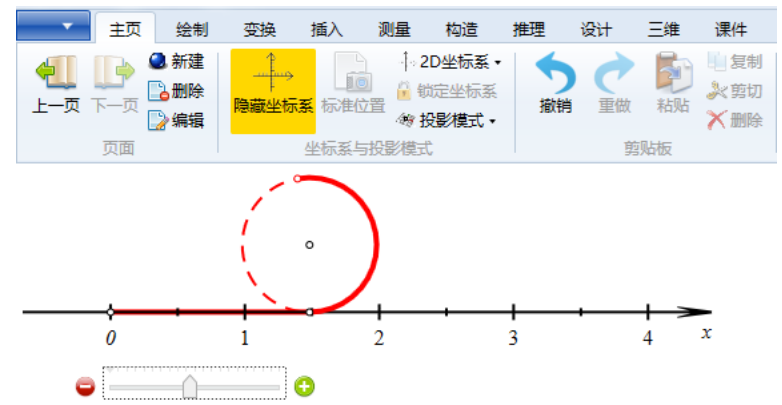

Figure 7. Draw parametric curve

\section{Designing the teaching of "circle"}

4.1. Perceiving the circle and introducing the new lesson

In life, we can often see the image of "circle." Teachers can naturally introduce new lesson content by showing pictures containing the image of "circle," such as the sun and the full moon.

\subsection{Taking a look back at history and asking questions}

Based on the application of the circle in the mathematics history of circle, the teacher introduces the history of "circle" to students and asks questions: What is a circle? What is the definition of circle?

\subsection{Inspiring thinking using historical materials}

Regarding the definition of the circle: more than 2,000 years ago, the Chinese philosopher Mozi gave circle a definition in the Mojing, which states that "a circle has the same length as one middle." Please think about the meaning of this sentence.

\subsection{Displaying historical materials and promoting understanding}

Borrowing from the "Circle Definition" product to display dynamically, not only can the length of the circle to the center of the circle be equalized, but also the process of a moving point rotating around the center of the circle can be dynamically displayed.

\subsection{Relating and understanding $\pi$}

Further relate $\pi$ to the "circle," and use the "circle cutting technique" and " $\pi$ " products made by Hawgent for dynamic display. The dynamic display shows that when the number of sides of a regular polygon inscribed in a circle increases indefinitely, the circumference is closer to the circumference. This process dynamically portrays the $\pi$ on the number axis, making it more intuitive to understand the $\pi$.

\subsection{Sublimating recognition and perceiving charm}

Combining the modern life application of "circle" and the implied meanings of fairness and perfection, it sublimates the understanding of "circle," realizes the beauty of "circle," and demonstrates the charm of culture.

\section{Conclusion}

\subsection{Analyzing the contents and uncovering the history of mathematics}

First of all, the textbook is an important material for teachers' teaching, but most of the content in the textbook does not clearly indicate the relevant background of the history of mathematics. When conducting HPM teaching supported by Hawgent, teachers should thoroughly analyze the content of the textbook and dig out the background of related content in the history of mathematics. This is the basis for the products 
of Hawgent and an important part of HPM teaching.

\subsection{Making the products and showing the history of mathematics}

Secondly, using Hawgent to make dynamic products for the excavated history of mathematics, but not all the content of the history of mathematics needs to be produced. On the one hand, it is necessary to judge whether it is suitable for dynamic display of products according to the characteristics of the content of the history of mathematics. In this process, it is also necessary to consider that the purpose of dynamic products is to make the content of the history of mathematics more dynamic, vivid, and efficient.

\subsection{Designing the teaching and integrating the history of mathematics}

Finally, the dynamic products of the history of mathematics produced by Hawgent is designed for the teaching process. In the design, new course content is naturally introduced on the basis of life; historical materials are used cleverly and reasonably integrated into the history of mathematics; the Hawgent product is used to dynamically display the history of mathematics. This is to promote more dynamic and vivid understanding in students, show the beauty of mathematics, and highlight the educational value of the history of mathematics.

\section{Acknowledgments}

The author would like to acknowledge Qian Tao, who participated in the study, for her instructive advice and useful suggestions in the writing of this paper. Many thanks for her help and encouragement.

\section{Disclosure statement}

The authors declare that there is no conflict of interest.

\section{Author contributions}

L.H. contributed to the conception and practice of the study. Q.T. helped perform the analysis with constructive discussions.

\section{References}

[1] Wang X, 2018, HPM Research Journey. Primary School Teaching (Mathematics Edition), (Z1): 4-9, 2.

[2] Gan C, Zuo C, Tang J, 2019, Hawgent Dynamic Mathematics Software Learning Topic: Quick Start. Friends of Mathematics, (03): 69-71.

[3] Wang S, 2016, Two Dimensions of Mathematical Infiltration of Aesthetic Education. Sichuan Education, (Z2): 37.

[4] Li P, 2016, Deep Experience, Deep Thinking, and Deepening Recognition - Teaching Thinking and Practice of "The Recognition of Circle". Primary School Teaching (Mathematics Edition), (10): 34-7.

[5] Fan Z, 2017, Form a Circle Without Center by Measuring. Mathematics Teaching and Learning in Junior Middle School, (03): 17-8.

[6] Zhang J, 2010, The Connotation of Circle Cutting and its Value in Mathematics Teaching. Mathematics Bulletin, (10): 19-22. 